\title{
10-YEAR EXPERIENCE WITH PATIENT-SPECIFIC MIDFACIAL RECONSTRUCTION - A SINGLE-CENTER STUDY
}

\author{
Aala'a Emara*, Asmaa Helmy**, Yasmine Nassar*, \\ Adel AbouElFetouh* and Maha Hakam*
}

\begin{abstract}
Complex midfacial reconstructions have greatly improved in quality with the introduction of computer aided/guided surgeries, 3D printing and Patient-specific implants (PSI). Our study reports a 10-year follow up of midfacial reconstruction patients receiving different PSI for reconstruction.

Methodology: During the period between 2011-2020 patients with midfacial defects requiring reconstruction with PSI were pooled together and the data analysed. The CTs, intraoperative notes and postoperative follow up notes were gathered. All surgeries were planned in a similar manner with the differences being in the material used and extents of defects; titanium PSIs for defects with alveolar segment involvement and Poly-ether-ether-ketone (PEEK) onlay for defects with zygomatic involvement causing cheek prominence deficiencies.
\end{abstract}

Results: Although intraoperative positioning was accurate when compared to the virtual planning in all our patients, several of them suffered soft tissue dehiscence specially those receiving Titanium PSIs.

Conclusions: From our experience PEEK seems to be more versatile and suitable for maxillofacial reconstructions but will need further modifications in methods of printing and reinforcement. Further research with larger sample sizes and different fabrication methods are needed to assess the techniques further.

KEYWORDS: Midfacial; patient-specific reconstruction; titanium, maxillofacial, PEEK, PSI, maxilla, zygoma

\section{INTRODUCTION}

Midfacial bony defects' reconstruction is an area of vast debate and extensive research. The sequelae of these defects include maxillomandibular dysfunction, impaired facial symmetry and unacceptable aesthetics ${ }^{[1]}$. Management of such defects aimed primarily to restore the function while achieving the best facial aesthetics possible. These defects are caused by oncologic resection or traumatic bone loss and can either be treated early or late. Midfacial defects in particular are of

\footnotetext{
* Faculty of Dentistry, Department of Oral \& Maxillofacial Surgery, Cairo University, Egypt

** Faculty of Dentistry, Department of Oral \& Maxillofacial Surgery, Ahram Canadian University, Egypt
} 
peculiar anatomy; difficult surgical access to the extensions and close proximity to vital structures make it challenging.

The treatment options for these complex defects dependes on bone grafting - autogenous or alloplastic grafts ${ }^{[2]}$. These have numerous complications including graft failure, infection, dehiscence, donor site infection \& scarring, increased morbidity and poor facial esthetics ${ }^{[3]}$. With the introduction of CAD/CAM (computer aided design/ computer aided manufacturing) technologies their use alone or in association with intraoperative navigational tools have been advantageous ${ }^{[4]}$. Although navigation tools are very pricey and not accessible to many surgical facilities, CAD/CAM technologies have evolved and become affordable to hospitals to research centres to invest in ${ }^{[5,6]}$. These technologies reduce intraoperative time, surgical morbidity, and greatly improve facial esthetics ${ }^{[7-9]}$. Their application includes fabrication of customized intraoperative templates, printed physical models (by mirroring the non-defective side) for mesh / plate adaptation and fabrication of patient specific implants (PSI). PSIs have been used in orthopaedic reconstructions ${ }^{[10]}$ and craniofacial applications ${ }^{[7,11]}$. The use of PSIs reduces operative time, improves surgical outcome and guarantees better postoperative facial symmetry ${ }^{[11]}$. Several materials have been reported for the use in midfacial reconstruction; including Polyether ether ketone (PEEK) and titanium. These showed 3D printing / milling capability for the fabrication of PSIs. PEEK has the advantage of its biocompatibility and allows addition of reinforcing materials; glass and carbon fibers ${ }^{[12-14]}$. It was specifically used in complex three dimensional cranial defects following craniectomy ${ }^{[15-18]}$. Patient-specific PEEK implants were used to reconstruct cases with residual maxillofacial defects and showed no complications within the 2-3 year follow-up ${ }^{[19-23]}$. The main advantage of PEEK was the low cost and easy milling/printing possibilities.

Moreover, titanium alloys have shown biocompatibility and promising results in several craniofacial applications ${ }^{[24-27]}$. Recently patient-specific implants were widely applied in craniofacial regions including eminence prosthesis ${ }^{[24]}$ and mandibular reconstruction devices ${ }^{[22,28]}$. The use of patient specific implants for midfacial defects was reported in combination with a vascularized flap ${ }^{[29]}$, other than that it has not been largely found in literature. This article aims to report the use computer aided/guided surgical techniques in midfacial reconstruction procedures in a single center across a 10 -year period.

\section{MATERIAL AND METHODS}

The study was designed to retrospectively assess the use of computer guided surgery / computer aided manufacturing technologies for midfacial reconstruction procedures during the period from 20112020. The cases included orbito-zygomatic defects, maxillary and midfacial defects requiring immediate or delayed reconstruction. The ethical committee of the Faculty of Dentistry Cairo University approved the study and all the patients consented to participation after presenting to the outpatient clinic at the OMFS unit. Faculty of Dentistry Cairo University.

All patients included in this study followed a similar preoperative assessment protocol of history taking, clinical examination, primary alginate impressions and CT scans. A numerical rating score [NRS] (0-10) was recorded for the patients to assess satisfaction with facial esthetics/symmetry. Preoperative DICOM files were imported into Materialise* software for analysis and presurgical planning.

\section{A) Treatment planning}

Surgical planning was performed on the Materialise software. The maxillary / midfacial bones were separated from the rest of the virtual

\footnotetext{
* Mimics 19.01 for intel X86 platform V15.0.1.7 CMaterialise n.v. Technologielaan 15, BE-3001 Leuven
} 
skull model (thresholding). The defective side is reconstructed by applying the mirror-image tool of the normal side onto the defect then a subtraction process was used to end up with just the necessary reconstruction/onlay part. Polyplanes were used to separate the designed reconstruction part from the adjacent bones and enable its fabrication. (fig 1)

Seven of our cases had orbitozygomatic traumatic defects causing facial asymmetry and were prepared to receive onlay PEEK implants. The 3 larger oncologic defects encompassing the maxilla were to receive 3D titanium PSIs with implant studs for future prosthetic rehabilitation.

\section{B) PSI design and fabrication}

\section{i) PEEK implant fabrication}

The onlay PEEK implants were designed by injection molding technique. Heated PEEK pellets (Bredent) ${ }^{*}$ were injected into the moulds of the designed reconstruction onlays using Thermopress machine ${ }^{* *}$ and left to harden. The final implant was deflasked and finished. Intraoperatively, maxillary vestibular transconjunctival approaches were used to expose the defects and place the implants which were fixed in place using 2-4 titanium miniscrews of $2.0 \mathrm{~mm}$ diameter (Figure 2).

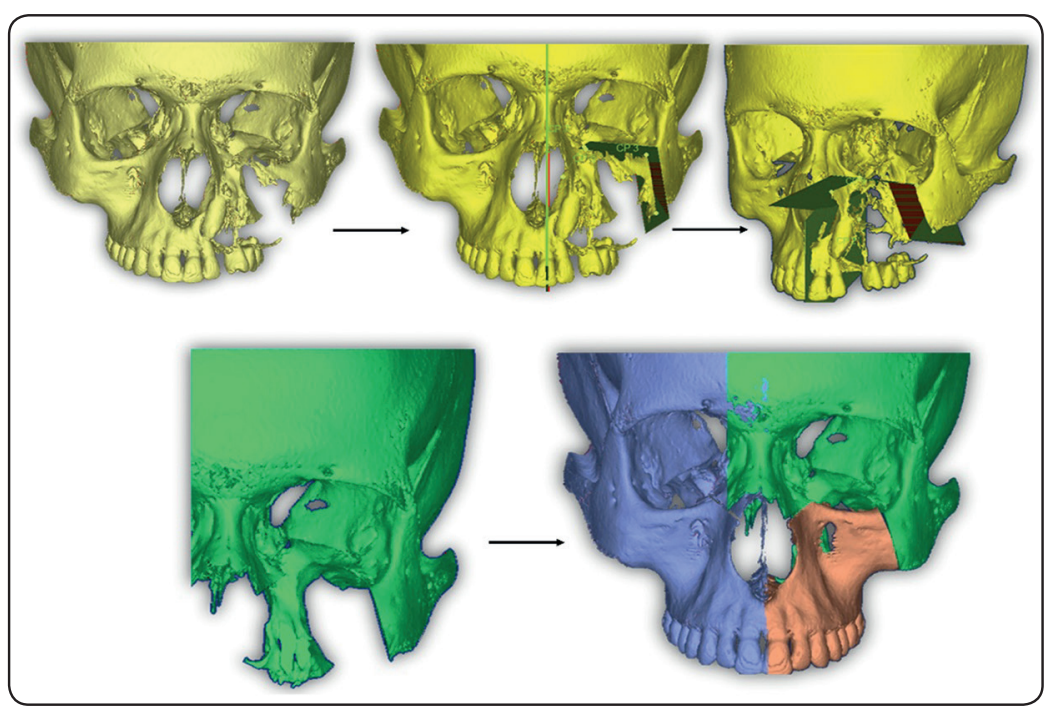

Fig. (1): Stages of preoperative planning and mirrorimaging of the normal side onto the defect
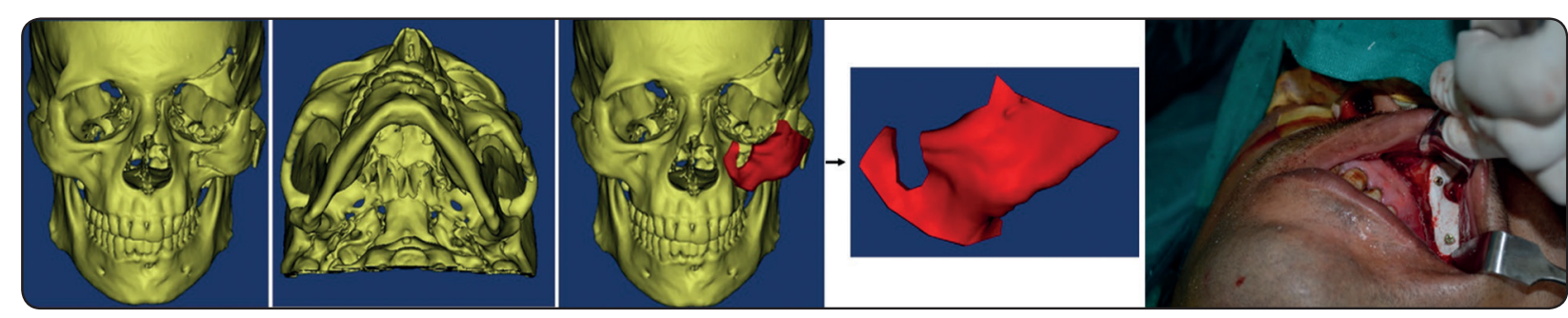

Fig. (2): Steps of design of PEEK onlay and its fixation intraoperatively.

GmbH \& Co.KG·Weissenhorner Str. 2·89250 Senden·Germany,www.bredent.com· @: info@bredent.com SHERAeco-mill 50 


\section{ii) Patient-specific titanium midface implants}

The midfacial post-oncologic defect cases were arranged to receive a titanium PSI made from a medical grade titanium material. The design of the PSI followed a similar procedure; importing of the CT scans into the software, a segmentation process to isolate the midfacial region, fabrication of an osteotomy guide and mirror-imaging of the normal side onto the defective side. An additional step of adding threaded implant like studs to the PSI and abutment like extensions for future prosthetic rehabilitation were added. Moreover the whole PSI was hollowed to reduce overall weight. The design included fixation studs on the palatal and zygomatic extensions to fix the PSI in place. Intraoperatively, an intraoral and an orbital approach were used to set the PSI in place.

The PSI was fabricated by an Electron Beam melting technology using Ti-6Al-4V ELI Titanium alloy Powder (Titanium $90 \%$, aluminum 6\% and vanadium 4\%) of particles size 45-100 $\mu \mathrm{m}$. (Figure 3).

\section{Post-operative followup}

All cases included in the study were covered by the native oral mucosa- primary intention healing with 3-0 vicryl resorbable suture material- except for a single delayed midfacial reconstruction case (patient \# 2) that received a radial forearm flap due to insufficient native soft tissue. Routine postoperative care was prescribed (antiobiotics, analgesics and oral hygiene instructions).

Postoperative CTs were ordered with the same specifications as the preoperative one and both superimposed to assess the facial symmetry. NRS scores were recorded after 6 weeks when complete healing was achieved.

These results were saved for further assessment.

\section{RESULTS}

Ten patients were included in this study; 7 with unilateral delayed post traumatic zygomatic defects and 3 with post-resection midfacial defects. Their ages ranged from15-60 years with a mean of 33.4 years old. Time lapsed from trauma/postoncologic defect to reconstruction ranged from 6-15 months with a mean of 11.29 months. Table 1 shows the defects, reconstruction plans and surgical approaches used.

All cases were operated on using intraoral incisions only except patient 2 where an already existing orbital scar was used to access the orbital wall along with the intraoral approach. An adequate number of screws could be placed to secure the implants to the native bone (2-4 screws) in all of the cases and stability was confirmed. Moreover, soft tissue was always adequate for proper 1ry closure over the implants; figure 4 shows one of our cases with the pre and postoperative CT cuts and clinical photos denoting the improvement of zygomatic prominence.

The titanium PSIs used were also fixed in place palatally and zygomatically using 3-5 miniscrews according to the size of the device. One patient had complications requiring plastic surgery to cover the exposed PEEK part with a rotation skin flap. On the other hand,
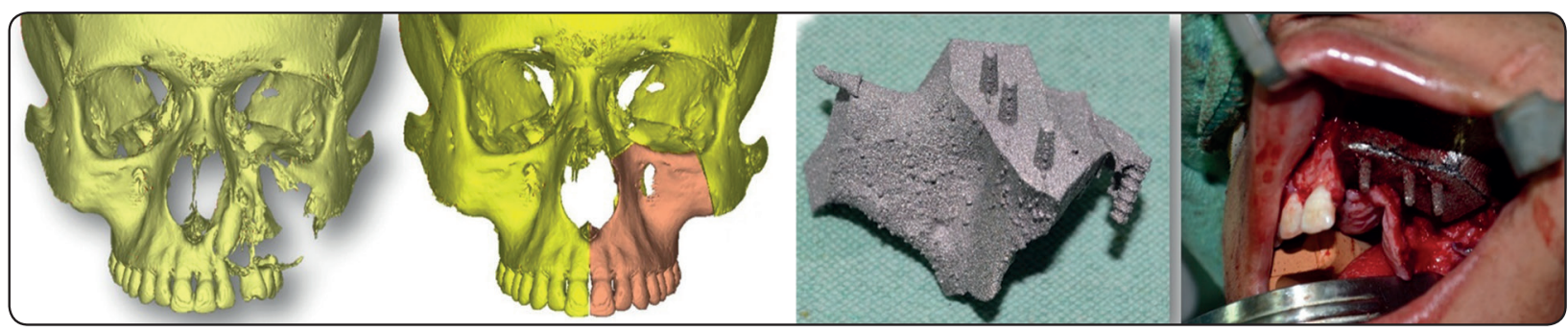

Fig. (3): Steps of fabrication of titanium PSI for one of our cases with the implant studs designed as shown to allow for future prosthetic rehabilitation. 
the titanium reconstruction cases showed mucosal dehiscence which was managed conservatively, and prosthetic rehabilitation accomplished.

Superimposition of postoperative CT images onto the planned preoperative ones showed anteroposterior deviation mean of $7.59 \mathrm{~mm}$ and an accuracy of $92.4 \%$ while the mediolateral deviation had a mean of $4.87 \mathrm{~mm}$ and an accuracy of $95.1 \%$. This was assessed by comparing the postoperative CTs by superimposition of the images.

TABLE (1): Patient surgical data; defects requiring reconstruction, types of reconstruction and approaches to be used

\begin{tabular}{|c|c|c|c|}
\hline Patient & Site of defect & Reconstruction plan & Surgical approach \\
\hline 1 & Isolated zygomatic defect & PEEK onlay through & intraoral approach \\
\hline 2 & Orbitozygomatic defect & PEEK onlay & $\begin{array}{l}\text { Intraoral approach and orbital } \\
\text { access through existing scar }\end{array}$ \\
\hline 3 & Isolated zygomatic defect & PEEK onlay & intraoral approach \\
\hline 4 & Post-oncologic resection of Lt maxilla & Titanium PSI with implant studs & intraoral approach \\
\hline 5 & Unicystic ameloblastoma of Rt maxilla & Titanium PSI & intraoral approach \\
\hline 6 & Isolated zygomatic defect & PEEK onlay & intraoral approach \\
\hline 7 & Isolated zygomatic defect & PEEK onlay & intraoral approach \\
\hline 8 & Orbitozygomatic defect & PEEK onlay & intraoral approach \\
\hline 9 & Isolated zygomatic defect & PEEK onlay & intraoral approach \\
\hline 10 & Gorlin's tumour - Rt maxilla & Titanium PSI with implant studs & intraoral approach \\
\hline
\end{tabular}
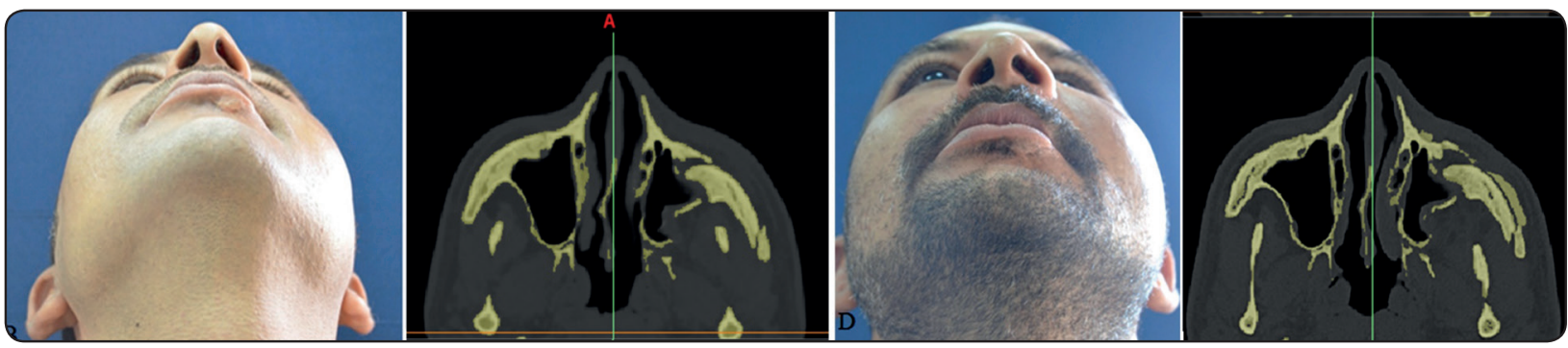

Fig. (4) : Showing one of the PEEK unlay implant patients with the clinical photos showing the improved zygomatic prominence clinically and on the CT (below: postoperative)
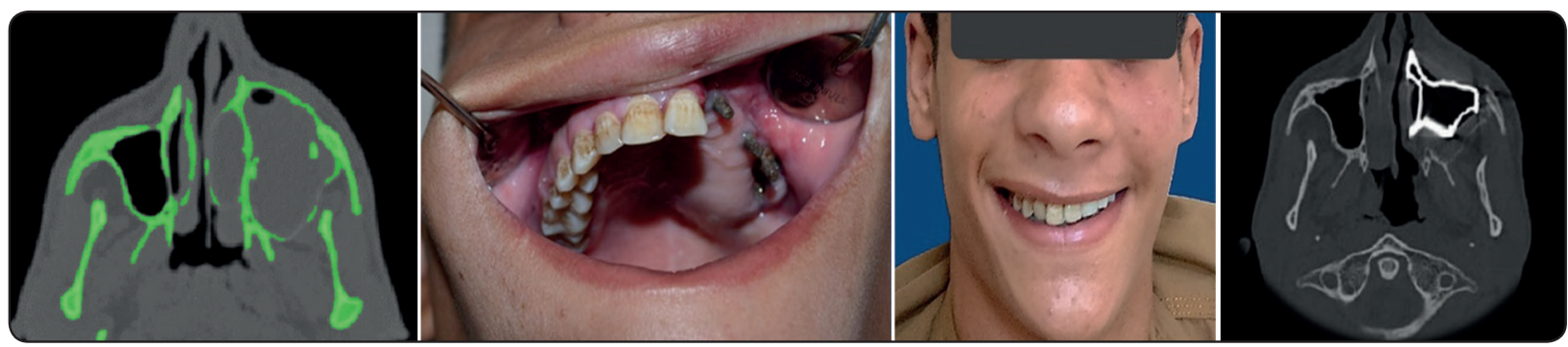

Fig. (5) : Shows the preoperative CT and postoperative clinical photos and CT of one of a patient treated with a Titanium PSI 
The NRS showed a significant difference in all cases with all 10 patients noting a satisfaction at the postoperative appearance. Throughout the followup period the patients attended mainly for touchups of their prosthetic appliances only.

\section{DISCUSSION}

Midfacial surgical reconstruction procedures usually include complex grafting procedures . Free flaps, pedicled faps and vascular flaps have all been reported for such cases with varying degrees of success. All these procedures carry an added cost of hospital stay, higher mortality/morbidity rates and higher costs ${ }^{[30]}$. With the rapidly evolving computer assisted designing, printing and surgery; their application in the field of maxillofacial reconstruction has also greatly developed. Many research groups have used several techniques to plan, design and perform surgeries using virtual models, cutting guides, positioning guides and so on. These reports have all concluded the superiority of the CAS groups in terms of accuracy and intraoperative time ${ }^{[31]}$.

In our study the defects we planned and reconstructed were all in the midface region whether posttraumatic/ post oncologic resection. The defects as reported earlier were either treated by patientspecific onlay PEEK implants or patient-specific titanium implants respectively. Titanium and PEEK are the only materials reported to be customized and used for midfacial reconstruction so far. Considered a promising material in complex maxillofacial reconstruction specifically in a study assessing its use in a series of orbitomaxillary and orbitocranial defects. Our study the use of PEEK for the onlay cases was due to the fabrication technique since the PSI were injection moulded or milled out of PEEK blocks. Its mechanical and chemical properties and comparably low cost supported its use as an onlay in the midface but would not justify its use in loadbearing regions as the maxillary alveolar process . It could be customized easily with good mechanical and chemical properties. On the other hand, in our cases with extensive maxillary-midfacial defects extending to the maxillary alveolar bone; titanium
PSIs were chosen as these would be fabricated using Electron beam melting technology to accommodate the complex 3D structure of the maxilla and allow for internal hollowing to mimic the sinus and reduce overall weight of the implants whilst being of sdequate biomechanical strength to withstand masticatory forces.

The PEEK used in the present study was reinforced with ceramic fillers to allow postoperative radiographic assessment of the PSI. they were fabricated using injection molding or milling depending on the availability of suitable milling machine and/or the PEEK blocks in addition to the size of the implant. The implants fabricated using injection moulding required slight intraoperative modficiation to allow accurate placement. The titanium PSIs moreover were fabricated using Electron Beam melting technique and required no intraoperative modification.

The postoperative CTs of the ten cases in this study proved proper placement and fixation of the implants. The titanium implants showed soft tissue dehiscence postoperatively that was treated later on according to the case. The PEEK onlays showed dehiscence in only one of the cases and a rotation flap was used to cover the implants.

This study reported the long term follow-up of Computer guided reconstructions of complex midfacial defects. The different reconstructing techniques depend on the size, complexity and location of the needed reconstruction implant. From our experience although the titanium PSIs were more versatile to fabricate and could easily be used in load-bearing areas; PEEK showed better soft tissue response and has better future perspectives with some additives and 3D fabrication technologies.

\section{CONCLUSIONS}

Reports on long term followups of novel reconstruction techniques are highly needed to assess the longevity of such reconstruction devices. The use of titanium is now becoming less appealing to surgeons due to multiple issues including soft 
tissue response, device cost and complexity as was noted in this study. Moreover PEEK seems to be the material of the future with the evolution of methods to print and modify it for better physical and biomechanical properties.

\section{DISCLOSURE STATEMENTS}

The authors report no conflicts of interest related to this study.

\section{REFERENCES}

1. Carr RM, Mathog RH. Early and delayed repair of orbitozygomatic complex fractures. J Oral Maxillofac Surg. 1997;55(3):253-8; discussion 8-9.

2. MATTHEW ECA, HANASONO. State-of-the-art reconstruction of midface and facial deformities. Journal of Surgical Oncology. 2016.

3. Moreno MA, Skoracki RJ, Hanna EY, Hanasono MM. Microvascular free flap reconstruction versus palatal obturation for maxillectomy defects. Head Neck. 2010; 32(7):860-8.

4. He Y, Zhu HG, Zhang ZY, He J, Sader R. Three-dimensional model simulation and reconstruction of composite total maxillectomy defects with fibula osteomyocutaneous flap flow-through from radial forearm flap. Oral Surg Oral Med Oral Pathol Oral Radiol Endod. 2009;108(6):e6-12.

5. Gruss JS, Van Wyck L, Phillips JH, Antonyshyn O. The importance of the zygomatic arch in complex midfacial fracture repair and correction of posttraumatic orbitozygomatic deformities. Plast Reconstr Surg. 1990; 85(6):878-90.

6. Wilde F, Lorenz K, Ebner AK, Krauss O, Mascha F, Schramm A. Intraoperative imaging with a 3D C-arm system after zygomatico-orbital complex fracture reduction. J Oral Maxillofac Surg. 2013;71(5):894-910.

7. Oh JH. Recent advances in the reconstruction of craniomaxillofacial defects using computer-aided design/computer-aided manufacturing. Maxillofac Plast Reconstr Surg. 2018;40(1):2.

8. Bell RB, Markiewicz MR. Computer-assisted planning, stereolithographic modeling, and intraoperative navigation for complex orbital reconstruction: a descriptive study in a preliminary cohort. J Oral Maxillofac Surg. 2009;67(12):2559-70.
9. Nassar Y, Barakat A, Hakam M, Abdel-Ghany K, AbouElFetouh A. Conventional versus computer-assisted techniques for reconstruction of orbitozygomatic fractures: a controlled clinical trial. International Journal of Oral and Maxillofacial Surgery. 2013;42(10).

10. Haglin JM, Eltorai AE, Gil JA, Marcaccio SE, BoteroHincapie J, Daniels AH. Patient-Specific Orthopaedic Implants. Orthop Surg. 2016;8(4):417-24.

11. Huang MF, Alfi D, Alfi J, Huang AT. The Use of PatientSpecific Implants in Oral and Maxillofacial Surgery. Oral Maxillofac Surg Clin North Am. 2019;31(4):593-600.

12. Schmidlin PR, Stawarczyk B, Wieland M, Attin T, Hammerle $\mathrm{CH}$, Fischer J. Effect of different surface pretreatments and luting materials on shear bond strength to PEEK. Dent Mater. 2010;26(6):553-9.

13. Dodier P, Winter F, Auzinger T, Mistelbauer G, Frischer JM, Wang WT, et al. Single-stage bone resection and cranioplastic reconstruction: comparison of a novel softwarederived PEEK workflow with the standard reconstructive method. Int J Oral Maxillofac Surg. 2020;49(8):1007-15.

14. Wang A, Lin R, Stark C, Dumbleton JH. Suitability and limitations of carbon fiber reinforced PEEK composites as bearing surfaces for total joint replacements. Wear. 1999;225-229:724-7.

15. Coulter C, Richards R, Peterson D, Collier J. Parietal skull reconstruction using immediate PEEK cranioplasty following resection for craniofacial fibrous dysplasia. J Plast Reconstr Aesthet Surg. 2014;67(8):e208-9.

16. Stawarczyk B, Beuer F, Wimmer T, Jahn D, Sener B, Roos $\mathrm{M}$, et al. Polyetheretherketone-a suitable material for fixed dental prostheses? J Biomed Mater Res B Appl Biomater. 2013;101(7):1209-16.

17. Jalbert F, Boetto S, Nadon F, Lauwers F, Schmidt E, Lopez R. One-step primary reconstruction for complex craniofacial resection with PEEK custom-made implants. J Craniomaxillofac Surg. 2014;42(2):141-8.

18. O'Reilly EB, Barnett S, Madden C, Welch B, Mickey B, Rozen S. Computed-tomography modeled polyether ether ketone (PEEK) implants in revision cranioplasty. J Plast Reconstr Aesthet Surg. 2015;68(3):329-38.

19. Kim MM, Boahene KD, Byrne PJ. Use of customized polyetheretherketone (PEEK) implants in the reconstruction of complex maxillofacial defects. Arch Facial Plast Surg. 2009;11(1):53-7. 
20. Guevara-Rojas G, Figl M, Schicho K, Seemann R, Traxler $\mathrm{H}$, Vacariu A, et al. Patient-specific polyetheretherketone facial implants in a computer-aided planning workflow. J Oral Maxillofac Surg. 2014;72(9):1801-12.

21. Berrone M AC, Pentenero M, Berrone S. Correction of a mandibular asymmetry after fibula reconstruction using a custom-made polyetheretherketone (PEEK) onlay after implant supported occlusal rehabilitation. Acta Otorhinolaryngol Ital. 2015 Oct;35(4):285-8.

22. Staal F, Pluijmers B, Wolvius E, Koudstaal M. Patient-Specific Implant for Residual Facial Asymmetry following Orthognathic Surgery in Unilateral Craniofacial Microsomia. Craniomaxillofac Trauma Reconstr. 2016; 9(3):264-7.

23. Hussain RN, Clark M, Berry-Brincat A. The Use of a Polyetheretherketone (PEEK) Implant to Reconstruct the Midface Region. Ophthalmic Plast Reconstr Surg. 2016;32(6):e151-e3.

24. Ihab R, Mounir R, Mounir M. Patient-specific titanium onlay eminoplasty: A novel protocol for treatment of recurrent temporomandibular joint dislocation. Int $\mathrm{J}$ Med Robot. 2020;16(4):e2114.

25. U V, Mehrotra D, Howlader D, Singh PK, Gupta S. Patient Specific Three-Dimensional Implant for Reconstruction of Complex Mandibular Defect. J Craniofac Surg. 2019;30(4):e308-e11.
26. Zhang WB, Yu Y, Mao C, Wang Y, Guo CB, Yu GY, et al. Outcomes of Zygomatic Complex Reconstruction With Patient-Specific Titanium Mesh Using Computer-Assisted Techniques. J Oral Maxillofac Surg. 2019;77(9):1915-27.

27. Jie B, Yao B, Li R, An J, Zhang Y, He Y. Post-traumatic maxillofacial reconstruction with vascularized flaps and digital techniques: 10-year experience. Int J Oral Maxillofac Surg. 2020;49(11):1408-15.

28. Mounir M, Abou-ElFetouh A, ElBeialy W, Mounir R. Patient-specific alloplastic endoprosthesis for reconstruction of the mandible following segmental resection: A case series. J Craniomaxillofac Surg. 2020; 48(8):719-23.

29. Mertens C, Lowenheim H, Hoffmann J. Image data based reconstruction of the midface using a patient-specific implant in combination with a vascularized osteomyocutaneous scapular flap. J Craniomaxillofac Surg. 2013; 41(3):219-25.

30. Emara A, ElFetouh AA, Hakam M, Mostafa B. Midfacial Reconstruction - A Systematic Review. Open Access Maced J Med Sci. 2016;4(3):468-75.

31. van Baar GJC, Schipper K, Forouzanfar T, Leeuwrik L, Winters HAH, Ridwan-Pramana A, et al. Accuracy of Computer-Assisted Surgery in Maxillary Reconstruction: A Systematic Review. J Clin Med. 2021;10(6). 